\title{
Design of Shaped Beam Planar Arrays of Waveguide Longitudinal Slots
}

\author{
Giovanni Andrea Casula, Giuseppe Mazzarella, and Giorgio Montisci \\ Dipartimento di Ingegneria Elettrica ed Elettronica, Università di Cagliari, Piazza d’Armi, 09123 Cagliari, Italy \\ Correspondence should be addressed to Giuseppe Mazzarella; mazzarella@diee.unica.it
}

Received 26 October 2012; Revised 10 January 2013; Accepted 21 January 2013

Academic Editor: Sembiam R. Rengarajan

Copyright ( 2013 Giovanni Andrea Casula et al. This is an open access article distributed under the Creative Commons Attribution License, which permits unrestricted use, distribution, and reproduction in any medium, provided the original work is properly cited.

\begin{abstract}
The Elliott's procedure for the design of a pencil beam waveguide longitudinal slot array has been generalized to encompass the design of shaped beam planar slot arrays. An extended set of design equations, taking into account in an operative way the feeding part of the array, has been devised. From this set of equations, a general and effective design procedure has been set up, shedding light on the constraints posed by a complex aperture distribution. The results of the proposed synthesis procedure have been validated through comparison with a commercial FEM software.
\end{abstract}

\section{Introduction}

Planar arrays of waveguide slots (Figure 1) have a very long story, since their use dates back at least from the 40s [1], and are still a very popular choice for high-performance antenna systems, especially in the higher part of microwave range [2]. Therefore, their pros and cons from both the mechanical and the electromagnetic point of view are well known [3]. The main advantages of these antennas are the high efficiency, the polarization purity [2-4], a considerable mechanical strength, a small size, and the great accuracy achievable both in the design and in the realization phase. The above features make the use of these antennas an effective solution in a wide range of applications [2], such as radar, aerospace, and satellite antennas. The most common drawbacks of waveguide slot arrays are the high realization cost, the small useful bandwidth, and the lack of flexibility; since once the array is realized, its electromagnetic behavior cannot be changed (though some countermeasures are possible $[5,6]$ ).

Many slot array configurations are available [2, 7-15], differing for slot type, slot pattern, and feeding architecture.

The most popular slot array configuration is the resonant array of longitudinal shunt slots [1], where the slots spacing is half the guided wavelength (at the center or "resonant" frequency) in the slotted waveguide. Therefore, we consider here this kind of array, which allows quite general feeding architectures. A single array (or subarray) consists of $M$ parallel-slotted waveguides (called radiating waveguides), with a transverse feeding guide, as in Figure 1. The feeding and radiating guides are coupled using $M$ series-series slots. Such slots are still spaced half the guided wavelength in the feeding waveguide, which is, therefore, the array spacing in the $E$-plane. Since the array bandwidth depends on the array size, a popular solution is to divide large arrays into subarrays, each one with its own matched input port. In this case, a beam-forming network (BFN) is required to properly feed the subarrays. A (quadrantal) subarray architecture is required also for monopulse radar antennas.

A slot array design splits naturally into an "internal" problem and an "external" one. The former is the physical design of the array which realizes an aperture distribution fulfilling the array pattern requirements, and whose input port is well matched. The latter is the computation of this aperture distribution, fulfilling the constraints imposed by the slot array technology.

The most accurate model describing the behavior of a resonant, pencil beam, and array of longitudinal slots has been proposed by Elliott in [16]. Elliott's work [16] is renowned since a highly accurate model of a slot array including mutual coupling is set there for the first time. Furthermore, it contains a second, less developed though equally important, point. As a matter of fact, the last section of [16] suggests that 


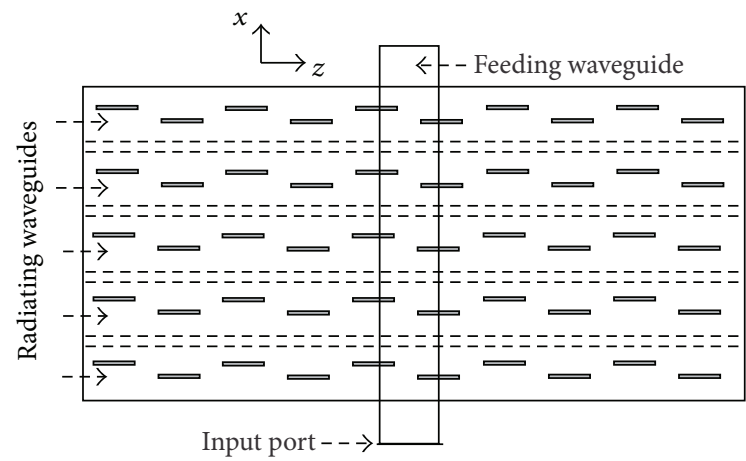

FIGURE 1: Geometry of an array or subarray.

the system of nonlinear design equations of a slot array can be solved in a very effective (and physically meaningful) way, evaluating all mutual couplings on the data of the previous iterative steps, since in this way the nonlinear equations for each slot can be decoupled. Actually, the decoupling details are not described in [16], also because they strongly depend on the array specifications. As a matter of fact, Elliott himself partly developed in [17, eq. (56)-(58)] the decoupling details for a planar equiphase array, but without taking into account the feeding network. After that, useful remarks on large arrays with a pencil beam are reported in [18], and some results on shaped beam arrays [19] have been reported for the first time in $[20,21]$, where the array is designed using an optimization technique, and, subsequently, in [22], using the Elliott's synthesis procedure. Unfortunately, the design procedures described in [20-22] have been applied by the authors only to linear arrays, but a large number of practical applications call for planar shaped beam arrays. As a matter of fact, most of the antennas used for satellite applications, radar systems, aerospace applications, and telecommunication systems are usually designed to produce a shaped beam in order to illuminate a selected geographical area with a maximum gain. These antennas require a complex aperture distribution, with a phase distribution spanning up to $360^{\circ}$.

The design of a planar array is a completely different matter compared to the design of a linear array. Actually, in the planar case the radiating slots interact both through the external mutual coupling and through the complex feeding network. Moreover, since the array geometry is not separable, the design of a shaped beam array is significantly different from the design of an array with equiphase slot voltages.

To the best of our knowledge, a complete synthesis procedure for a planar waveguide slot array with complex distribution has not been described in international literature. Aim of this paper is to fill this gap providing an effective and accurate design technique for a shaped beam planar array, which takes into account not only the external mutual coupling, but also the strong slot interaction due to the feeding network.

The first problem to face in the design of a waveguide slot array with a shaped beam pattern is that the excitation phase of each slot cannot span $360^{\circ}$. A very limited solution is to realize a shaped beam array with real excitations, as in [23], but the results are not satisfactory. On the other hand, array pattern synthesis procedures, able to take into account excitations constraints, have been proposed [24], and these procedures could be exploited by suitably limiting the amplitude and phase variation of the slot excitations, in order to get an aperture distribution achievable with a waveguide slot array.

In this work, we propose an "internal" design procedure for shaped beam planar arrays of waveguide longitudinal slots by extending Elliott's model and devising from it a new synthesis procedure. This is not straightforward, since the original Elliott's equations [17] must be properly modified to take into account a further degree of freedom, namely, the phase of the slot excitations. In Sections 2 and 3, the new derived design equations and the array synthesis procedure are described in detail. In Section 4, this procedure has beenvalidated and tested, in a way independent of the Elliott's model, using a commercial FEM solver, namely, HFSS 13 by Ansoft. The results obtained with this FEM CAD are in very good agreement with experimental results, as reported in the open literature for a wide range of applications (see, e.g., $[25,26])$.

A number of shaped beam arrays with different patterns have been designed, and two of them are discussed in detail. The analysis performed with HFSS shows that the presented examples fulfill the required specifications.

\section{Design Equations for Slot Arrays}

The behavior of a planar slot array is ruled by a set of design equations, linking the electrical variables of the array to the geometrical ones. These equations describe

(1) the slot excitation due to the radiating guide [16];

(2) the external mutual coupling between the slots [16];

(3) the interaction between the radiating slots due to the BFN [27-29].

The interaction (3) is strongly frequency dependent. Therefore, since a resonant array is always designed at the center frequency, only the corresponding equations at this frequency will be used in this work.

We consider here a planar array composed by $M$ radiating waveguides, each one carrying a, possibly different, number $N_{m}$ of radiating slots. The first radiating slot of the $m$ th radiating waveguide is indicated by $\widehat{I}_{m}$, and the numbering proceeds arbitrarily from right to left, while $\widehat{N}_{m}$ and $\widehat{F}_{m}$ denote, respectively, the slot immediately to the right of the feeding coupling slot and the last slot of the $m$ th radiating waveguide, as shown in Figure 2.

This numbering allows to design arrays with regular or irregular aperture shapes in the same way. In this reference system, the array E-plane is vertical, and the axis of the radiating waveguides is horizontal. Each slot is completely characterized by its length and its offset with respect to the waveguide axis, which is assumed positive upward.

An array of longitudinal slots can also be divided into subarrays (as in the example in Figure 3). Each subarray is made of a number of radiating waveguides, fed by a feeding 


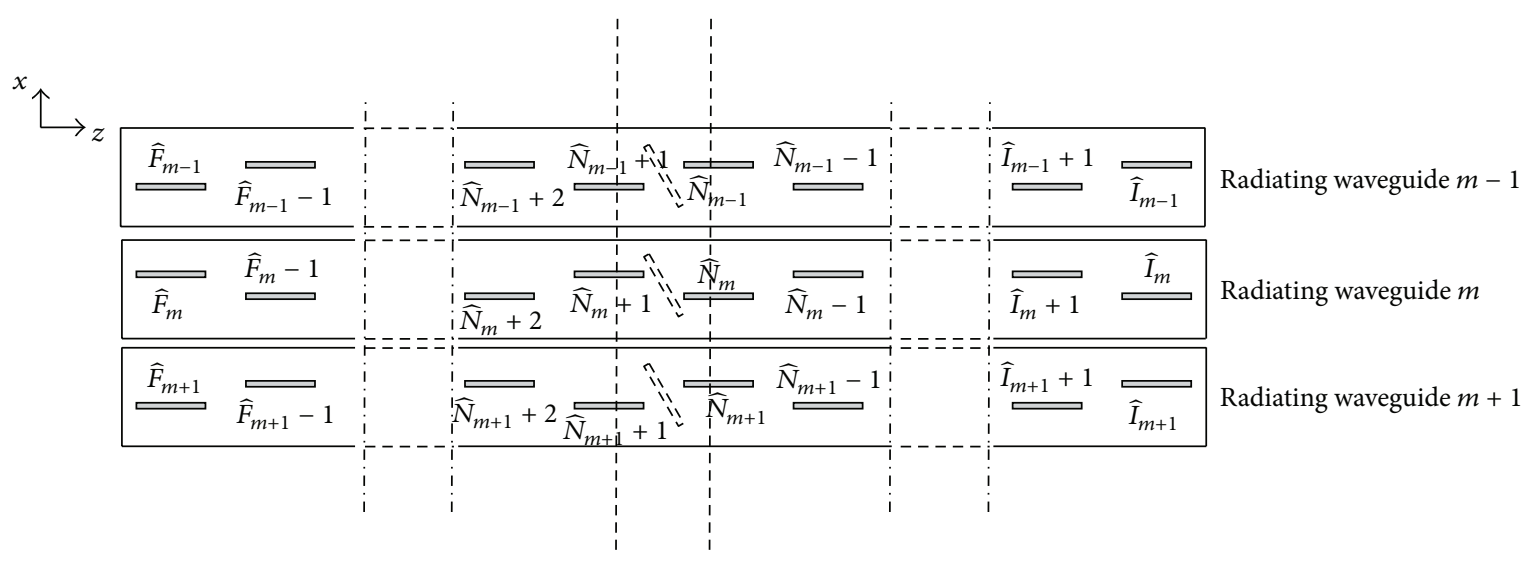

FIGURE 2: Radiating slots numbering.

waveguide (orthogonal to the radiating ones) through a sequence of series-series inclined coupling slot (one for each radiating waveguide of the subarray) [30]. Each feeding waveguide is then fed at its input node through a seriesseries inclined coupling slot. All the coupling slots have been chosen resonant. Therefore, a generic array is composed by $N$ radiating slots, $M$ radiating waveguides, and $Q$ subarrays and has consequently $Q$ feeding waveguides and $Q$ input ports. In the example shown in Figure 3(a), the array is divided into 4 subarrays. Each subarray is composed by 4 radiating waveguides, and the design procedure allows each radiating waveguide to contain a different number of radiating slots. Figure 3(b) shows the four waveguides, each one feeding a subarray, and the input port is shown for each subarray.

Let $v_{m}$ be the $\left(\mathrm{TE}_{10}\right.$ fundamental) mode voltage on the $m$ th radiating waveguide. The array design equations can be written taking into account that the mode voltage $V_{n}$ at the position of the $n$th radiating slot is different in each radiating waveguide and can be written as $V_{n}=(-1)^{n-\widehat{I}_{m}} v_{m}$. Therefore, the first two sets of design equations for a slot array are [17]

$$
\begin{gathered}
\frac{Y_{n}^{A}}{G_{R}}=j K_{1} \tilde{f}_{n} \sigma_{n} \frac{V_{n}^{S}}{V_{n}} \\
=j K_{1} \widetilde{f}_{n} \sigma_{n} \frac{V_{n}^{S}}{(-1)^{n-\widehat{I}_{m}} v_{m}}, \\
\frac{Y_{n}^{A}}{G_{R}}=\frac{2 \sigma_{n}^{2}}{D_{n}},
\end{gathered}
$$

wherein the $\left\{V_{n}^{S}\right\}$ are the slot excitations required by the aperture distribution, and

$$
\begin{gathered}
K_{1}=-\frac{2}{\beta_{10}} \cdot \frac{\pi}{a} \cdot \sqrt{\frac{2}{(k \cdot a)(k \cdot b)}}, \\
\sigma_{n}=\sin \frac{\pi x_{n}}{a}, \\
\tilde{Y}_{n}=\frac{Y_{n}}{\sigma_{n}^{2}},
\end{gathered}
$$

$$
\begin{gathered}
\widetilde{f}_{n}=\widetilde{f}\left(l_{n}\right)=\frac{\left(\pi / 2 k l_{n}\right) \cos \beta_{10} l_{n}}{\left(\pi / 2 k l_{n}\right)^{2}-\left(\beta_{10} / k\right)^{2}}, \\
R_{n}=j \alpha \sum_{\substack{j=1 \\
j \neq n}}^{N} g_{n j} \frac{V_{j}^{S}}{V_{n}^{S}}, \\
D_{n}=\frac{2}{\widetilde{Y}_{n} / G_{R}}+\frac{1}{\widetilde{f}_{n}^{2}} R_{n},
\end{gathered}
$$

wherein $a$ and $b$ are the waveguide transverse dimensions, $k$ is the wavenumber in free space, $G_{R}$ and $\beta_{10}$ are the equivalent admittance and the propagation constant of the $\mathrm{TE}_{10}$ fundamental waveguide mode, and $Y_{n}, l_{n}$, and $x_{n}$ are, respectively, the self-admittance, the length, and the offset of the $n$th slot of the array.

In (6), $g_{n j}$ is the sum of the external coupling between the radiating slots [16] and of the internal coupling due to the interaction between the radiating slots through higher-order waveguide modes [31].

At the input node of the $m$ th radiating waveguide (Figure 4), the inclined coupling slot feeding the waveguide is modeled (being resonant) as an ideal transformer, with a current transformation ratio equal to $C_{m}[30,32-34]$.

The input impedance seen at the input of this series-series transformer is therefore

$$
Z_{m}=\frac{C_{m}^{2}}{G_{R}} \sum_{n=\widehat{I}_{m}}^{\widehat{F}_{m}} \frac{Y_{n}^{A}}{G_{R}} .
$$

Since the mode voltages $v_{m}$ are not independent for radiating waveguides fed by the same feeding waveguide, we must take into account the equations of the feeding line. Also the feeding waveguide is fed by a series-series transformer, with known input current. Subsequent equations will be more clear if we consider each half of the feeding guide as a separate guide. With this convention, two feeding waveguides are represented in Figure 5, namely, the $q$ th and $(q+Q)$ th waveguides. The index $q$ assumes therefore the values between 1 and $Q$, where $Q$ represents the number of the feeding waveguides of the array (namely, the number 


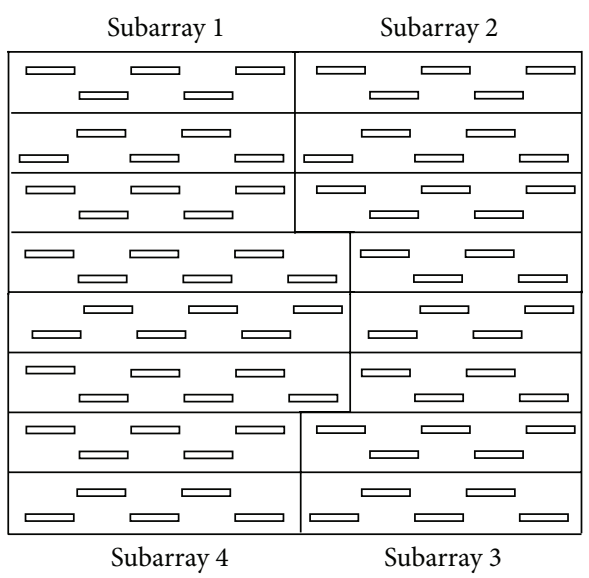

(a)

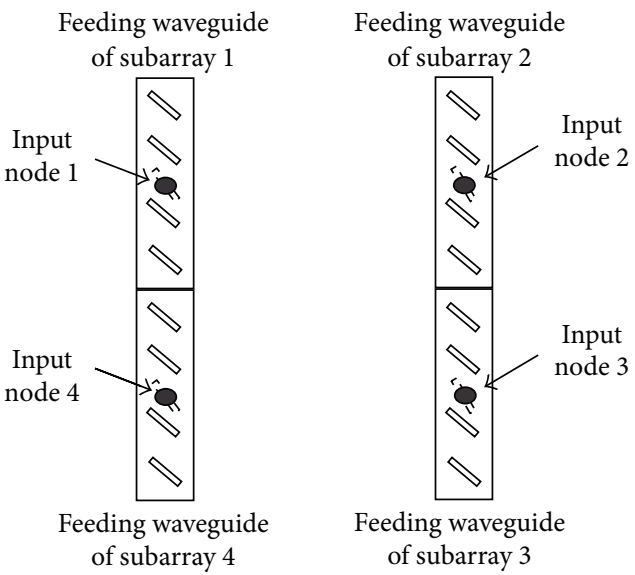

(b)

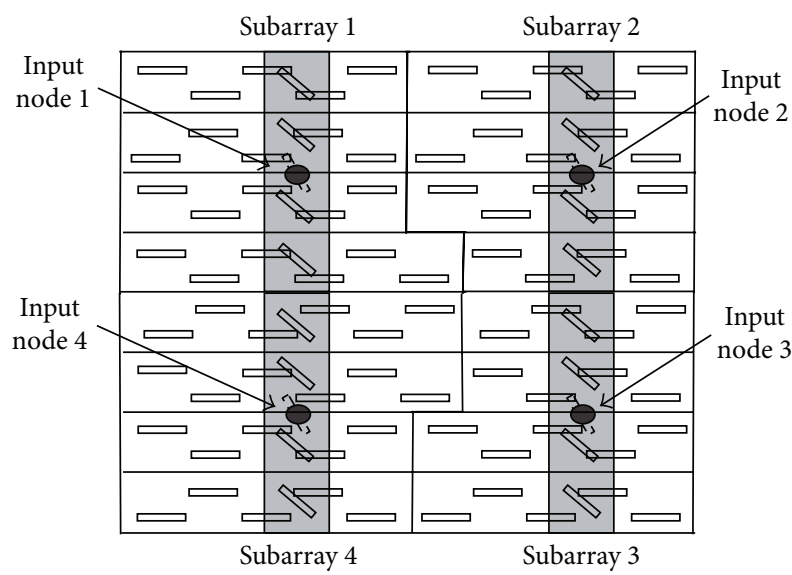

(c)

FIGURE 3: (a) An example of subarrays structure; (b) feeding waveguides and input ports; (c) complete array.

of input ports of the array). Let $\widetilde{I}_{q}$ be the current flowing into the last coupling slot of the $q$ th feeding waveguide (the farther from the feeding node), having the same direction of $I_{q}$ (see Figure 5). This coupling slot feeds the first radiating waveguide, which we denote by $\widehat{P}_{q}$. The current flowing on the

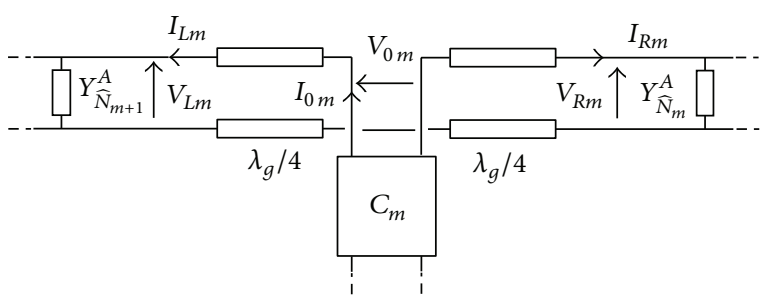

FIGURE 4: Input node of the $m$ th radiating waveguide. The two admittances model the radiating slots $\widehat{N}_{m}$ (on the right hand) and $\widehat{N}_{m+1}$ (on the left hand).

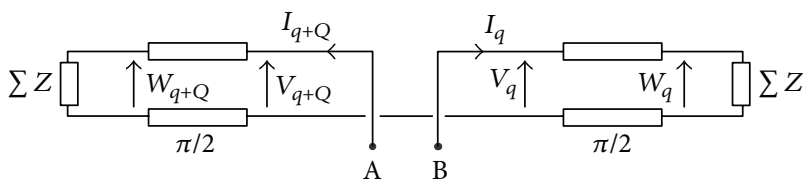

FIGURE 5: Input node of the feeding waveguide.

subsequent coupling slots will be $(-1)^{m-\widehat{P}_{q}} \widetilde{I}_{q}$, where $\widehat{P}_{q}+1 \leq$ $m \leq \widehat{M}_{q}$, and $\widehat{M}_{q}$ represents the coupling slot corresponding to the last radiating waveguide, fed by the $q$ th feeding guide.

The current $I_{0 m}$ (with $m=\widehat{P}_{q}$ ) flowing on the first radiating waveguide (see Figure 4 for the generic $m$ th radiating guide) fed by the $q$ th feeding guide is therefore given by $C_{m} \widetilde{I}_{q}$, while $C_{m}(-1)^{m-\widehat{P}_{q}} \widetilde{I}_{q}$ is the current flowing on the other radiating waveguides, with $\widehat{P}_{q}+1 \leq m \leq \widehat{M}_{q}$.

The mode voltage at the slot $\widehat{N}_{m}$ (which, as shown in Figure 4 , is the radiating slot immediately at the right of the coupling slot feeding the $m$ th radiating waveguide) is then given by

$$
\begin{aligned}
V_{R, m} & =-\frac{j}{G_{R}}\left(-I_{0, m}\right) \\
& =C_{m}(-1)^{m-\widetilde{P}_{q}} \widetilde{I}_{q} \frac{j}{G_{R}} .
\end{aligned}
$$

Since (from Figure 2) $V_{\widehat{N}_{m}}=V_{R, m}=(-1)^{\widehat{N}_{m}-\widehat{I}_{m}} v_{m}$, the mode voltage on the $m$ th radiating waveguide can be expressed as

$$
\begin{aligned}
v_{m} & =(-1)^{\widehat{N}_{m}-\widehat{I}_{m}} V_{\widehat{N}_{m}}=(-1)^{\widehat{N}_{m}-\widehat{I}_{m}} V_{R, m} \\
& =\left[C_{m}(-1)^{m-\widehat{P}_{q}} \widetilde{I}_{q} \frac{j}{G_{R}}\right](-1)^{\widehat{N}_{m}-\widehat{I}_{m}} .
\end{aligned}
$$

Finally, as indicated in Figure 5, the input impedance at the port $A B$ is given by

$$
\begin{array}{r}
G_{A} Z_{q}^{\mathrm{IN}}=\frac{1}{G_{A} \sum_{[q]} Z_{m}}+\frac{1}{G_{A} \sum_{[q+Q]} Z_{m}}, \\
q=1, \ldots, Q,
\end{array}
$$

where the notations $[q],[q+Q]$ indicate that the sums are extended to all the radiating waveguides fed by the $q$ th and 
$(q+Q)$ th feeding waveguides, respectively (see Figure 5); $G_{A}$ is the equivalent admittance of the $\mathrm{TE}_{10}$ fundamental mode in the feeding waveguide.

\section{Synthesis Procedure}

In order to design a slot array, we have to solve the nonlinear systems (1) and (11), which require an iterative solution.

The input data of the design procedure are the radiating slot excitations (namely, the $N$ slot voltages $V_{n}^{S}$ ) and the input impedances $Z_{q}^{\mathrm{IN}}$ at each input node of the array. The procedure gives as output the lengths and offsets of all the radiating slots.

Following Elliott suggestion [16], it is convenient to evaluate the mutual coupling coefficients $R_{n}$, given by (6), using the data of the previous iterative step, since small changes in offsets and lengths cause only a small change in the mutual coupling. With this choice, the equations are decoupled, and it is possible to recompute the new parameters of each slot independently of the other slots.

A shaped beam array requires a complex aperture distribution, therefore the Elliott's design equations (1) must be properly modified because a further set of requirements, the phase of the slot excitations, must be taken into account. On the other hand, no further degrees of freedom are available, so a different strategy must be devised in order to extend the Elliott's procedure [16] to the shaped beam case.

Let

$$
V_{n}^{S}=\left|V_{n}^{S}\right| e^{j \varphi_{n}^{S}}
$$

the slot voltage of the $n$th radiating slot of the array.

A complex slot voltage distribution, such as (12), requires that some other electrical quantities are complex. Among them, there are the feeding currents $\widetilde{I}_{q}$. We include also a sign variable into their definition, which reads

$$
\begin{gathered}
\widetilde{I}_{q}=\left|\widetilde{I}_{q}\right| \exp \left(j \psi_{q}\right) S_{q}, \\
\widetilde{I}_{q+Q}=-\left|\widetilde{I}_{q+Q}\right| \exp \left(j \psi_{q+Q}\right) S_{q+Q},
\end{gathered}
$$

where $S_{q}=+/-1$ is a sign to be determined, and $\psi_{q}$ is defined by $\psi_{q}=\arctan \left(\operatorname{Im}\left[\widetilde{I}_{q}\right] / \operatorname{Re}\left[\tilde{I}_{q}\right]\right)$, so that $-\pi / 2 \leq \psi_{q} \leq \pi / 2$. With this choice, in the limit case of a complex distribution, but with all phases of the slot voltages equal to zero $\left(\varphi_{n}^{s}=0\right)$, we come back to the equiphase case, being $\widetilde{I}_{q}=\left|\widetilde{I}_{q}\right| S_{q}$ and $\psi_{q}=0$, without ambiguity.

Since $C_{m}$ is a real number we get, from (10)

$$
\begin{aligned}
v_{m}= & {\left[C_{m}(-1)^{m-\widehat{P}_{q}}\left|\widetilde{I}_{q}\right| \frac{j}{G_{R}}\right] } \\
& \times(-1)^{\widehat{N}_{m}-\widehat{I}_{m}} \exp \left(j \psi_{q}\right) S_{q} .
\end{aligned}
$$

As a consequence, all the mode voltages $v_{m}$ on the radiating waveguides fed by the same feeding waveguide (identified by the index $q$ ) are equiphase.
The active admittance, using (1), can therefore be expressed as

$$
\begin{aligned}
\frac{Y_{n}^{A}}{G_{R}}= & K_{1} \tilde{f}_{n} \sigma_{n} \frac{G_{R}\left|V_{n}^{S}\right|}{C_{m}\left|I_{q}\right|} \cdot(-1)^{\widehat{N}_{m}-n} \cdot(-1)^{m-\widehat{P}_{q}} \\
& \cdot \exp \left[j\left(\varphi_{n}^{S}-\psi_{q}\right)\right] S_{q}=\frac{2 \sigma_{n}^{2}}{D_{n}} .
\end{aligned}
$$

The input impedances $Z_{q}^{\mathrm{IN}}$ required at the feeding nodes of the array are real numbers in almost all practical applications. Therefore, it follows from (11) that $\left(\sum_{[q]} Z_{m}\right)^{-1}$ and $\left(\sum_{[q+Q]} Z_{m}\right)^{-1}$ must have an opposite imaginary part. As a consequence, the problem is not determined, and the simpler choice is to require that all the $Z_{m}$ have a real value.

Now, from (15), it follows that

$$
\operatorname{Im}\left\{D_{n} \exp \left[j\left(\varphi_{n}^{S}-\psi_{q}\right)\right]\right\}=0, \quad n=1, \ldots, N
$$

as also found in $[20,21]$, for the linear case $\left(\psi_{q} \equiv 0\right)$.

The left-hand side of (16) depends only on the slot length $l_{n}$, since the offsets are fixed to the values of the previous iterative step. Therefore, (16) is the sought equation for the new value of this length. If $\tilde{l}_{n}$ is the solution of (16), then

$$
\delta_{n}=\left\{D_{n} \exp \left[j\left(\varphi_{n}^{S}-\psi_{q}\right)\right]\right\}_{l_{n}=\tilde{l}_{n}}, \quad n=1, \ldots, N
$$

is real. Using (17) in (15), we obtain the following expression for the active admittances:

$$
\frac{Y_{n}^{A}}{G_{R}}=\frac{2 \sigma_{n}^{2}}{\delta_{n}} \exp \left[j\left(\varphi_{n}^{S}-\psi_{q}\right)\right], \quad n=1, \ldots, N .
$$

Then, comparing (18) with (15), we get

$$
\sigma_{n}=K_{1} \widetilde{f}_{n} \frac{\delta_{n}}{2} \frac{G_{R}\left|V_{n}^{S}\right|}{C_{m}\left|\widetilde{I}_{q}\right|}(-1)^{\widehat{N}_{m}-n}(-1)^{m-\widehat{P}_{q}} S_{q} .
$$

Finally, by replacing (19) in (15), the active admittance can be expressed as

$$
\frac{Y_{n}^{A}}{G_{R}}=\frac{\delta_{n}}{2} K_{1}^{2} \widetilde{f}_{n}^{2}\left[\frac{G_{R}\left|V_{n}^{S}\right|}{C_{m}\left|\widetilde{I}_{q}\right|}\right]^{2} \exp \left[j\left(\varphi_{n}^{S}-\psi_{q}\right)\right] .
$$

If we put in (8) the active admittances given by (20), the input impedance $Z_{m}$ seen at the primary of the feeding transformer can be written as

$$
\begin{aligned}
Z_{m}= & K_{1}^{2} \frac{G_{R}}{2} \frac{1}{\left|\widetilde{I}_{q}\right|^{2}} \cdot \sum_{n=\widehat{I}_{m}}^{\widehat{F}_{m}} \delta_{n} \widetilde{f}_{n}^{2}\left|V_{n}^{S}\right|^{2} \\
& \times \exp \left[j\left(\varphi_{n}^{S}-\psi_{q}\right)\right], \quad m=1, \ldots, M .
\end{aligned}
$$

Since the input node is a series one, the relation between the currents $\left|\widetilde{I}_{q}\right|$ and $\left|\widetilde{I}_{q+Q}\right|$ can be expressed as

$$
\frac{(-1)^{\widehat{M}_{q}-\widehat{P}_{q}} S_{q}}{T_{q}\left|\widetilde{I}_{q}\right|}=\frac{(-1)^{\widehat{M}_{q+Q}-\widehat{P}_{q+Q}} S_{q+Q}}{T_{q+Q}\left|\widetilde{I}_{q+Q}\right|}, \quad q=1, \ldots, Q,
$$


where

$$
\begin{aligned}
T_{q} & =\frac{1}{\sum_{[q]} \delta_{n} \tilde{f}_{n}^{2}\left|V_{n}^{S}\right|^{2} \exp \left(j \varphi_{n}^{S}\right)}, \\
T_{q+Q} & =\frac{1}{\sum_{[q+Q]} \delta_{n} \tilde{f}_{n}^{2}\left|V_{n}^{S}\right|^{2} \exp \left(j \varphi_{n}^{S}\right)} .
\end{aligned}
$$

Let $H_{q}^{q+Q}$ be a real positive parameter defined by

$$
\left|\widetilde{I}_{q}\right|=H_{q}^{q+Q} \cdot\left|\widetilde{I}_{q+Q}\right| \cdot
$$

Using $H_{q}^{q+Q}$, we can write

$$
T_{q} H_{q}^{q+Q}=T_{q+Q}(-1)^{\widehat{M}_{q}-\widehat{P}_{q}}(-1)^{\widehat{M}_{q+Q}-\widehat{P}_{q+Q}} S_{q} S_{q+Q} .
$$

From (25), it follows that $T_{q}$ and $T_{q+Q}$ must have the same phase (apart from the sign), once the convergence of the iterative design procedure has been reached.

Finally, we must fulfill the requirement on the input impedance $Z_{q}^{\mathrm{IN}}$ at the secondary of the transformer feeding the waveguides $q$ and $q+Q$. This impedance is the sum of the input impedances of the two waveguides:

$$
G_{A} Z_{q}^{\mathrm{IN}}=G_{A} Z_{q}^{\mathrm{eq}}+G_{A} Z_{q+Q}^{\mathrm{eq}}
$$

which are (see (11) and (21)) as follows:

$$
\begin{aligned}
G_{A} Z_{q}^{\mathrm{eq}}= & {\left[K_{1}^{2} \frac{G_{A} G_{R}}{2} \frac{1}{\left|\widetilde{I}_{q}\right|^{2}} \quad q=1, \ldots, Q,\right.} \\
& \left.\times \sum_{[q]} \delta_{n} \widetilde{f}_{n}^{2}\left|V_{n}^{S}\right|^{2} \exp \left[j\left(\varphi_{n}^{S}-\psi_{q}\right)\right]\right]^{-1}, \\
G_{A} Z_{q+Q}^{\mathrm{eq}}= & {\left[K_{1}^{2} \frac{G_{A} G_{R}}{2} \frac{1}{\left|\widetilde{I}_{q+Q}\right|^{2}} \quad\right.} \\
& \left.\times \sum_{[q+Q]} \delta_{n} \widetilde{f}_{n}^{2}\left|V_{n}^{S}\right|^{2} \exp \left[j\left(\varphi_{n}^{S}-\psi_{q}\right)\right]\right]^{-1} .
\end{aligned}
$$

Equations (27) can be simplified using (23) as follows:

$$
\begin{gathered}
G_{A} Z_{q}^{\mathrm{eq}}=\frac{2}{K_{1}^{2} G_{A} G_{R}}\left|\widetilde{I}_{q}\right|^{2} T_{q} \exp \left(j \psi_{q}\right), \\
G_{A} Z_{q+Q}^{\mathrm{eq}}=\frac{2}{K_{1}^{2} G_{A} G_{R}}\left|\widetilde{I}_{q+\mathrm{Q}}\right|^{2} T_{q+Q} \exp \left(j \psi_{q+Q}\right) .
\end{gathered}
$$

The input impedance $Z_{q}^{\mathrm{IN}}$ must have real and positive values, while $Z_{q}^{\text {eq }}$ and $Z_{q+Q}^{\text {eq }}$ can be real or complex. However, we have enough available degrees of freedom to force both $Z_{q}^{\mathrm{eq}}$ and
$Z_{q+Q}^{\mathrm{eq}}$ to have real and positive values. With this choice, we can fix the phases $\psi_{q}$ and $\psi_{q+Q}$ as follows:

$$
\begin{gathered}
\psi_{q}=-\arg \left(T_{q}\right), \\
\psi_{q+Q}=-\arg \left(T_{q+Q}\right) .
\end{gathered}
$$

The input impedance $Z_{q}^{\mathrm{IN}}$ can be finally expressed as

$$
\begin{aligned}
G_{A} Z_{q}^{\mathrm{IN}}= & \frac{2}{K_{1}^{2} G_{A} G_{R}} \\
& \times\left[\left|\widetilde{I}_{q}\right|^{2} T_{q} \exp \left(j \psi_{q}\right)+\left|\widetilde{I}_{q+Q}\right|^{2} T_{q+Q} \exp \left(j \psi_{q+Q}\right)\right] \\
= & \frac{2\left|\widetilde{I}_{q}\right|^{2}}{K_{1}^{2} G_{A} G_{R}}\left[\left|T_{q}\right|+\frac{\left|T_{q+Q}\right|}{\left(H_{q}^{q+Q}\right)^{2}}\right] .
\end{aligned}
$$

Equation (30) allows to determine $\left|\widetilde{I}_{q}\right|$ from the required value of $Z_{q}^{\mathrm{IN}}$, thus terminating the iterative step.

It is worth noting that, in order to avoid convergence problems, the initial values of $\psi_{q}$ must be properly connected to the values of the voltage distribution. Therefore, even in the first iterative step, the phases of the active admittances must be kept relatively small, avoiding problems of oscillating or trapped solutions.

The synthesis procedure proposed in this section has no limitations by itself, since it can design the array geometry for every aperture distribution that a slot array can radiate. On the other hand, the excitation phase achievable with a longitudinal radiating slot cannot span the whole $360^{\circ}$, but is limited to

$$
\begin{aligned}
& {\left[-\varphi_{\mathrm{MAX}} \leq \varphi \leq \varphi_{\mathrm{MAX}}\right] \cup[} {\left[\left(180^{\circ}-\varphi_{\mathrm{MAX}}\right)\right.} \\
&\left.\leq \varphi \leq\left(180^{\circ}+\varphi_{\mathrm{MAX}}\right)\right],
\end{aligned}
$$

where $\varphi_{\operatorname{MAX}}$ depends slightly on the waveguide dimensions but is always not larger than $60^{\circ}$. However, to prevent convergence problems, it can be safe to choose a smaller $\varphi_{\mathrm{MAX}}$, for example $50^{\circ}$.

As a consequence, an arbitrary voltage distribution cannot always be radiated by a slot array. However, since different aperture distributions can radiate essentially equivalent shaped patterns, this "hardware" limitation can be circumvented using array pattern design techniques which allow the introduction of appropriate constraints both for voltage amplitudes and phases (compare [24]). The amplitude constraints prevent the synthesis procedure to obtain too small slot lengths and/or offsets, while the phase constraints take into account the limited excitation phase that each slot can span.

\section{Results}

In order to assess the synthesis procedure, a number of planar arrays, with different size and aperture distributions, 


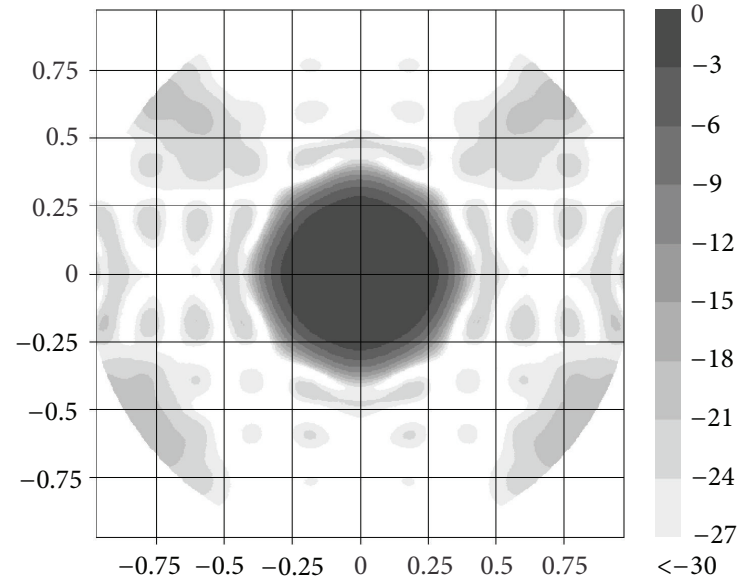

Figure 6: Contour plot ( $\mathrm{dB}$ scale) of the $8 \times 8$ planar array with a circular radiation pattern in the $u-v$ plane.

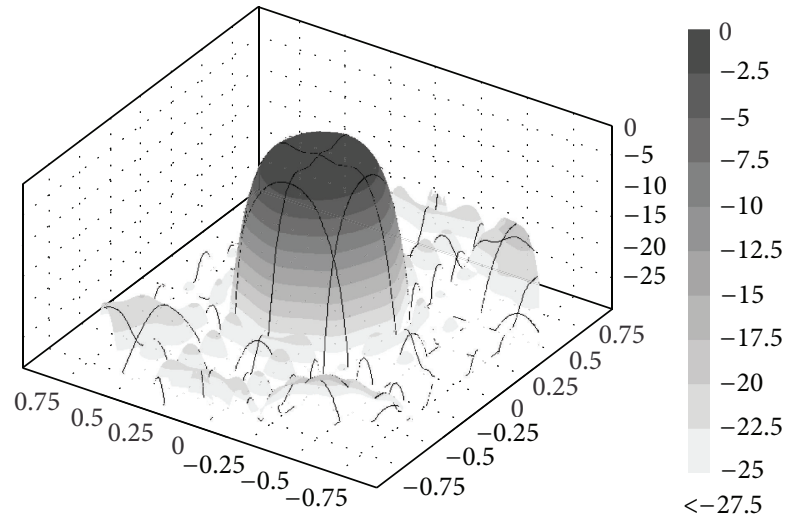

FIGURE 7: $3 \mathrm{D}$ radiation pattern ( $\mathrm{dB}$ scale) of the $8 \times 8$ planar array with a circular radiation pattern in the $u$ - $v$ plane.

have been designed with the procedure of Section 3. Inhouse softwares have been used to evaluate both the slot selfadmittance [35] and the mutual coupling [36, 37].

Once the geometry of the designed array has been determined, an analysis has been performed to check whether the array requirements are fulfilled. This has been done using both [28] and a commercial FEM solver, namely, HFSS. Since the former is based on Elliott's model, while the latter is independent of it and is considered essentially equivalent to experimental verification $[25,26]$, we present here only the HFSS results, which fulfill all the requirements and therefore fully assess our procedure. It is worth noting that the results obtained by the procedure of [28] are equivalent to the ones simulated with HFSS.

The architecture of the arrays presented in this section is shown in Figure 1, where both the radiating waveguides and the feeding waveguide are half-height WR90 waveguides $(22.86 \mathrm{~mm} \times 5.08 \mathrm{~mm})$ with $1 \mathrm{~mm}$ wall thickness. The feeding

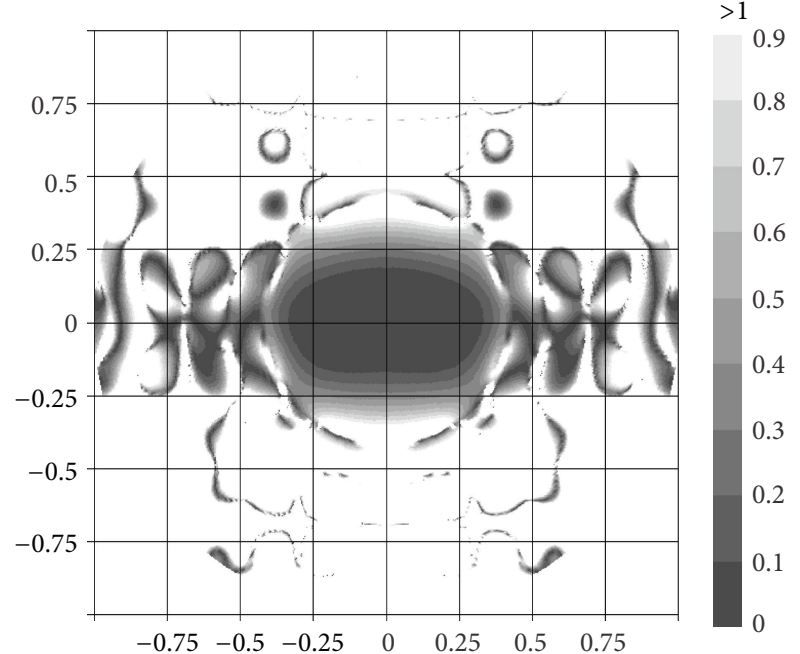

FIGURE 8: Differences between the required and designed radiation pattern (both in $\mathrm{dB}$ scale) for the $8 \times 8$ planar array with a circular radiation pattern in the $u-v$ plane.

waveguide has been fed at its side by a waveguide port, and the radiating waveguides have been fed through a seriesseries inclined resonant coupling slot. All the coupling slots have a length equal to $17.07 \mathrm{~mm}$, a width of $1.5 \mathrm{~mm}$, and the tilt angle with respect to the feeding waveguide axis is $45^{\circ}$, corresponding to a coupling coefficient equal to 1 (see $[30,32,33]$ for details).

Starting from a specified and arbitrary-shaped beam pattern, we have used the array patterns synthesis procedure described in [24] to compute the required excitations. According to the considerations made at the end of Section 3 about the excitations achievable with a longitudinal radiating slot, appropriate constraints both on the amplitude and on the phase of the slot excitations are required, in order to get an aperture distribution achievable with an array of slots. In particular, the maximum phase and the minimum normalized amplitude of the slot excitations have been set, respectively, to $\varphi_{\text {MAX }}=50^{\circ}$ and $\left|V_{S \text {, min }}\right|=0.1$.

In the first example, we present an $8 \times 8$ planar array, fed by a single feeding waveguide containing 8 coupling slots, designed requiring a circular pattern, with a radius equal to 0.25 in the $(u, v)$ plane, with $-20 \mathrm{~dB}$ sidelobes, and a ripple of $\pm 0.5 \mathrm{~dB}$. The normalized amplitudes and the phases of the required slot excitations are reported in Tables 1(a) and 1(b), respectively, while the corresponding designed slot lengths and offsets are shown in Tables $1(\mathrm{c})$ and $1(\mathrm{~d})$.

The contour plot of the simulated (HFSS) far field pattern for the designed array is shown in Figure 6. In Figure 7, the 3D far field pattern is depicted, and Figure 8 shows the difference between the pattern obtained using the required slot voltages and the designed pattern. In the shaped region, this difference is less than $0.3 \mathrm{~dB}$. Figure 9 shows an enlargement of the shaped region with a ripple of about $\pm 0.5 \mathrm{~dB}$. The frequency response of the array is shown in Figure 10, 


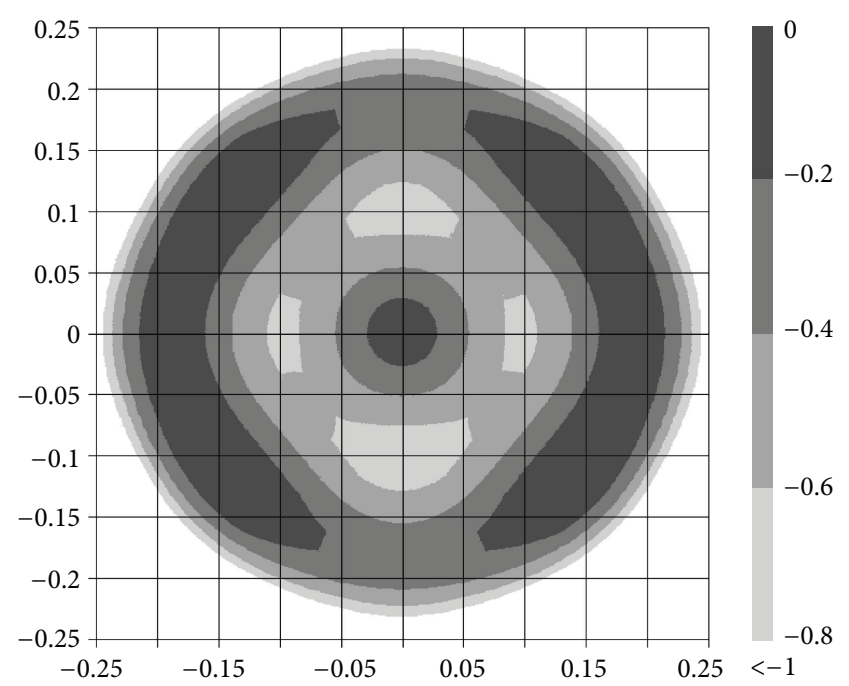

FIGURE 9: Ripple of the $8 \times 8$ planar array with a circular radiation pattern in the $u-v$ plane (expressed in $\mathrm{dB}$ ).

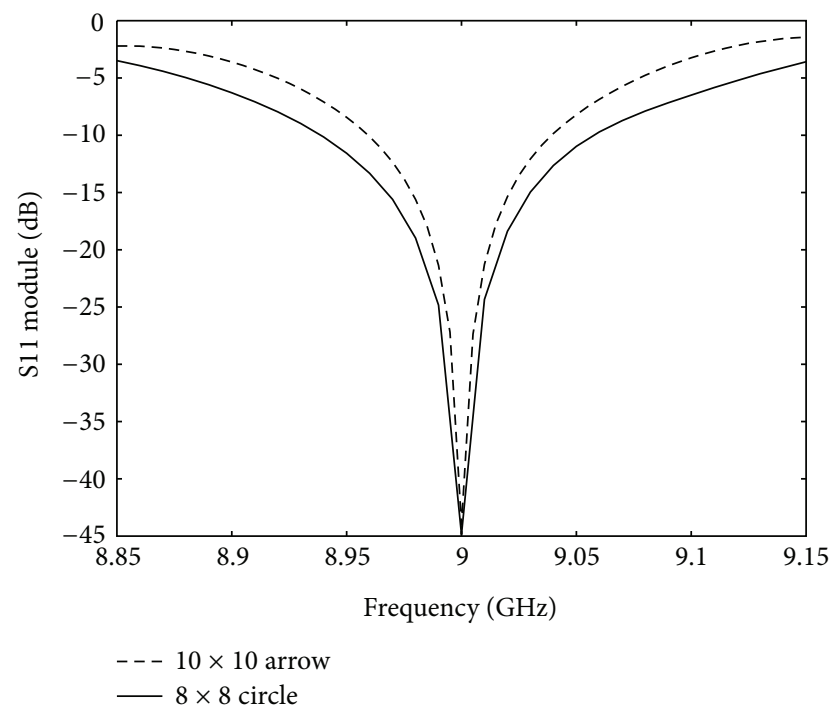

FIGURE 10: Simulated frequency responses (HFSS) of the designed arrays.

while Figures 11 and 12 show, respectively, the $E$-plane and the $H$-plane far field patterns within the working frequency bandwidth. The behaviour of the shaped radiation pattern is still very good even at the upper and at the lower ends of the bandwidth.

The second example is a $10 \times 10$ planar array, fed by a single feeding waveguide containing 8 coupling slots, designed requiring an arrow-shaped pattern (see the dashed line in Figure 16 for the required geometry), with $-15 \mathrm{~dB}$ sidelobes. The normalized amplitudes and the phases of the required slot excitations are reported in Tables 2(a) and 2(b), and the corresponding designed slot lengths and offsets are shown in Tables 2(c) and 2(d).
TABLE 1: (a) Required normalized amplitude of slots excitations for the $8 \times 8$ planar array with a circular radiation pattern. (b) Required phase (degrees) of slots excitations for the $8 \times 8$ planar array with a circular radiation pattern. (c) Designed slots lengths $(\mathrm{mm})$ for the $8 \times 8$ planar array with a circular radiation pattern. (d) Designed slots offsets $(\mathrm{mm})$ for the $8 \times 8$ planar array with a circular radiation pattern.

(a)

\begin{tabular}{lcccccccc}
\hline Radiat. & $\begin{array}{c}\text { Slot } \\
\text { waveg. }\end{array}$ & $\begin{array}{c}\text { Slot } \\
1\end{array}$ & $\begin{array}{c}\text { Slot } \\
3\end{array}$ & $\begin{array}{c}\text { Slot } \\
4\end{array}$ & $\begin{array}{c}\text { Slot } \\
5\end{array}$ & $\begin{array}{c}\text { Slot } \\
6\end{array}$ & $\begin{array}{c}\text { Slot } \\
7\end{array}$ & $\begin{array}{c}\text { Slot } \\
8\end{array}$ \\
\hline 1 & 0.10 & 0.10 & 0.15 & 0.16 & 0.16 & 0.15 & 0.10 & 0.10 \\
2 & 0.10 & 0.18 & 0.17 & 0.10 & 0.10 & 0.17 & 0.18 & 0.10 \\
3 & 0.15 & 0.17 & 0.19 & 0.49 & 0.49 & 0.19 & 0.17 & 0.15 \\
4 & 0.16 & 0.10 & 0.49 & 1.00 & 1.00 & 0.49 & 0.10 & 0.16 \\
5 & 0.16 & 0.10 & 0.49 & 1.00 & 1.00 & 0.49 & 0.10 & 0.16 \\
6 & 0.15 & 0.17 & 0.19 & 0.49 & 0.49 & 0.19 & 0.17 & 0.15 \\
7 & 0.10 & 0.18 & 0.17 & 0.10 & 0.10 & 0.17 & 0.18 & 0.10 \\
8 & 0.10 & 0.10 & 0.15 & 0.16 & 0.16 & 0.15 & 0.10 & 0.10 \\
\hline
\end{tabular}

(b)

\begin{tabular}{lcccccccc}
\hline Radiat. & Slot & Slot & Slot & Slot & Slot & Slot & Slot & Slot \\
waveg. & 1 & 2 & 3 & 4 & 5 & 6 & 7 & 8 \\
\hline 1 & 129.9 & 10.9 & 3.6 & 0.0 & 0.0 & 3.6 & 10.9 & 129.9 \\
2 & 10.9 & 6.5 & 19.3 & 47.9 & 47.9 & 19.3 & 6.5 & 10.9 \\
3 & 3.6 & 19.3 & 129.9 & 139.4 & 139.4 & 129.9 & 19.3 & 3.6 \\
4 & 0.0 & 47.9 & 139.4 & 149.1 & 149.1 & 139.4 & 47.9 & 0.0 \\
5 & 0.0 & 47.9 & 139.4 & 149.1 & 149.1 & 139.4 & 47.9 & 0.0 \\
6 & 3.6 & 19.3 & 129.9 & 139.4 & 139.4 & 129.9 & 19.3 & 3.6 \\
7 & 10.9 & 6.5 & 19.3 & 47.9 & 47.9 & 19.3 & 6.5 & 10.9 \\
8 & 129.9 & 10.9 & 3.6 & 0.0 & 0.0 & 3.6 & 10.9 & 129.9 \\
\hline
\end{tabular}

(c)

\begin{tabular}{lcccccccc}
\hline Radiat. & Slot & Slot & Slot & Slot & Slot & Slot & Slot & Slot \\
waveg. & 1 & 2 & 3 & 4 & 5 & 6 & 7 & 8 \\
\hline 1 & 16.04 & 15.58 & 15.82 & 16.24 & 16.24 & 15.82 & 15.58 & 16.04 \\
2 & 15.44 & 15.98 & 15.49 & 17.29 & 7.29 & 15.49 & 15.98 & 15.44 \\
3 & 16.23 & 14.82 & 16.17 & 16.27 & 16.27 & 16.17 & 14.82 & 16.23 \\
4 & 15.92 & 13.47 & 16.85 & 16.16 & 16.16 & 16.85 & 13.47 & 15.92 \\
5 & 15.89 & 13.17 & 16.33 & 16.34 & 16.34 & 16.33 & 13.17 & 15.89 \\
6 & 15.74 & 15.24 & 16.71 & 16.16 & 16.16 & 16.71 & 15.24 & 15.74 \\
7 & 16.28 & 15.60 & 15.63 & 7.31 & 17.31 & 15.63 & 15.60 & 16.28 \\
8 & 16.55 & 15.97 & 15.70 & 16.27 & 16.27 & 15.70 & 15.97 & 16.55 \\
\hline
\end{tabular}

(d)

\begin{tabular}{lcccccccc}
\hline Radiat. & Slot & Slot & Slot & Slot & Slot & Slot & Slot & Slot \\
waveg. & 1 & 2 & 3 & 4 & 5 & 6 & 7 & 8 \\
\hline 1 & -0.19 & -0.20 & 0.27 & -0.12 & 0.12 & -0.27 & 0.20 & 0.19 \\
2 & -0.15 & 0.26 & -0.33 & -0.21 & 0.21 & 0.33 & -0.26 & 0.15 \\
3 & 0.08 & -0.60 & -0.13 & 0.58 & -0.58 & 0.13 & 0.60 & -0.08 \\
4 & -0.29 & 0.86 & 0.67 & -1.05 & 1.05 & -0.67 & -0.86 & 0.29 \\
5 & 0.24 & -1.18 & -0.64 & 1.11 & -1.11 & 0.64 & 1.18 & -0.24 \\
6 & -0.20 & 0.49 & 0.32 & -0.48 & 0.48 & -0.32 & -0.49 & 0.20 \\
7 & 0.06 & -0.25 & 0.39 & 0.25 & -0.25 & -0.39 & 0.25 & -0.06 \\
8 & 0.30 & 0.21 & -0.25 & 0.12 & -0.12 & 0.25 & -0.21 & -0.30 \\
\hline
\end{tabular}

The contour plot of the simulated (HFSS) far field pattern for the designed array is shown in Figure 13. In Figure 14 
TABLE 2: (a) Required normalized amplitude of slots excitations for the $10 \times 10$ planar array with an arrow-shaped radiation pattern. (b) Required phase (degrees) of slots excitations for the $10 \times 10$ planar array with an arrow-shaped radiation pattern. (c) Designed slots lengths $(\mathrm{mm})$ for the $10 \times 10$ planar array with an arrow-shaped radiation pattern. (d) Designed slots offsets $(\mathrm{mm})$ for the $10 \times 10$ planar array with an arrow-shaped radiation pattern.

(a)

\begin{tabular}{|c|c|c|c|c|c|c|c|c|c|c|}
\hline Radiat. waveg. & Slot 1 & Slot 2 & Slot 3 & Slot 4 & Slot 5 & Slot 6 & Slot 7 & Slot 8 & Slot 9 & Slot 10 \\
\hline 1 & 0.229 & 0.224 & 0.206 & 0.143 & 0.100 & 0.100 & 0.110 & 0.211 & 0.238 & 0.206 \\
\hline 2 & 0.299 & 0.289 & 0.237 & 0.100 & 0.100 & 0.100 & 0.190 & 0.301 & 0.336 & 0.306 \\
\hline 3 & 0.249 & 0.235 & 0.102 & 0.100 & 0.198 & 0.323 & 0.438 & 0.467 & 0.390 & 0.306 \\
\hline 4 & 0.122 & 0.100 & 0.240 & 0.428 & 0.592 & 0.696 & 0.723 & 0.634 & 0.460 & 0.282 \\
\hline 5 & 0.237 & 0.285 & 0.525 & 0.764 & 0.926 & 1.000 & 0.969 & 0.791 & 0.549 & 0.310 \\
\hline 6 & 0.237 & 0.285 & 0.525 & 0.764 & 0.925 & 0.999 & 0.968 & 0.790 & 0.549 & 0.310 \\
\hline 7 & 0.120 & 0.100 & 0.241 & 0.429 & 0.591 & 0.695 & 0.721 & 0.633 & 0.459 & 0.281 \\
\hline 8 & 0.249 & 0.233 & 0.102 & 0.100 & 0.202 & 0.326 & 0.439 & 0.468 & 0.390 & 0.307 \\
\hline 9 & 0.299 & 0.287 & 0.234 & 0.100 & 0.100 & 0.100 & 0.190 & 0.301 & 0.336 & 0.305 \\
\hline 10 & 0.228 & 0.223 & 0.207 & 0.144 & 0.100 & 0.100 & 0.112 & 0.212 & 0.239 & 0.206 \\
\hline
\end{tabular}

(b)

\begin{tabular}{lcccccccccc}
\hline Radiat. waveg. & Slot 1 & Slot 2 & Slot 3 & Slot 4 & Slot 5 & Slot 6 & Slot 7 & Slot 8 & Slot 9 & Slot 10 \\
\hline 1 & 18.5 & 0.00 & -13.8 & -29.9 & 41.7 & 50.6 & 50.6 & 50.6 & 50.6 & 37.6 \\
2 & -25.9 & -24.6 & -28.0 & -32.0 & 50.5 & 50.6 & 50.6 & 50.6 & 50.6 & 50.6 \\
3 & -35.9 & -42.1 & -9.2 & 166.2 & -179 & 143.4 & 130.6 & 130.6 & 130.6 & 130.6 \\
4 & 38.6 & 175.7 & -171.9 & -178.7 & 173.6 & 164.2 & 155.3 & 149.0 & 145.2 & 148.3 \\
5 & 130.6 & 146.9 & 171.5 & 177.6 & 176.6 & 171.8 & 167.1 & 165.9 & 167.9 & -177 \\
6 & 130.6 & 147.3 & 171.7 & 177.7 & 176.6 & 171.8 & 167.1 & 165.8 & 167.8 & -177 \\
7 & 38.5 & 176.5 & -171.8 & -178.5 & 173.6 & 164.3 & 155.4 & 149.0 & 145.2 & 148.2 \\
8 & -35.4 & -41.7 & -8.9 & 166.0 & -179 & 144.0 & 130.6 & 130.6 & 130.6 & 130.6 \\
9 & -25.6 & -24.3 & -27.7 & -31.8 & 50.5 & 50.6 & 50.6 & 50.6 & 50.6 & 50.6 \\
10 & 18.3 & -0.21 & -14.1 & -30.0 & 41.5 & 50.6 & 50.6 & 50.6 & 50.6 & 38.6 \\
\hline
\end{tabular}

(c)

\begin{tabular}{lllllllllll}
\hline Radiat. waveg. & Slot 1 & Slot 2 & Slot 3 & Slot 4 & Slot 5 & Slot 6 & Slot 7 & Slot 8 & Slot 9 & Slot 10 \\
\hline 1 & 16.07 & 15.81 & 16.28 & 16.94 & 15.50 & 14.93 & 16.04 & 15.63 & 15.04 & 15.78 \\
2 & 16.47 & 16.09 & 16.24 & 17.86 & 15.17 & 15.37 & 15.75 & 15.81 & 15.44 & 14.94 \\
3 & 16.66 & 16.23 & 16.26 & 16.16 & 15.75 & 16.65 & 16.61 & 16.44 & 16.75 & 16.81 \\
4 & 14.96 & 15.95 & 16.12 & 16.10 & 16.18 & 16.23 & 16.38 & 16.41 & 16.27 & 16.77 \\
5 & 16.98 & 16.52 & 16.15 & 16.25 & 16.14 & 16.23 & 16.31 & 16.23 & 16.30 & 15.98 \\
6 & 17.04 & 16.38 & 16.29 & 16.13 & 16.24 & 16.20 & 16.26 & 16.35 & 16.13 & 16.20 \\
7 & 15.17 & 16.32 & 15.91 & 16.26 & 16.07 & 16.27 & 16.42 & 16.27 & 16.48 & 16.51 \\
8 & 16.42 & 16.44 & 15.84 & 15.74 & 15.94 & 16.55 & 16.58 & 16.60 & 16.49 & 17.13 \\
9 & 16.68 & 15.95 & 16.41 & 17.35 & 15.60 & 15.11 & 15.86 & 15.82 & 15.27 & 15.42 \\
10 & 15.82 & 15.99 & 16.17 & 17.08 & 15.24 & 15.20 & 15.92 & 15.55 & 15.24 & 15.41 \\
\hline & & & & & & & & & &
\end{tabular}

(d)

\begin{tabular}{lcccccccccc}
\hline Radiat. waveg. & Slot 1 & Slot 2 & Slot 3 & Slot 4 & Slot 5 & Slot 6 & Slot 7 & Slot 8 & Slot 9 & Slot 10 \\
\hline 1 & 0.19 & -0.13 & 0.21 & -0.15 & 0.10 & -0.21 & 0.11 & -0.22 & 0.40 & -0.28 \\
2 & -0.32 & 0.13 & -0.14 & 0.15 & -0.19 & 0.11 & -0.21 & 0.28 & -0.42 & 0.69 \\
3 & 0.29 & -0.14 & 0.03 & 0.08 & -0.07 & 0.31 & -0.42 & 0.29 & -0.41 & 0.39 \\
4 & -0.34 & -0.15 & 0.16 & -0.27 & 0.38 & -0.48 & 0.55 & -0.44 & 0.27 & -0.30 \\
5 & -0.34 & 0.22 & -0.36 & 0.45 & -0.66 & 0.72 & -0.69 & 0.58 & -0.38 & 0.30 \\
6 & 0.32 & -0.23 & 0.34 & -0.49 & 0.60 & -0.74 & 0.69 & -0.56 & 0.43 & -0.23 \\
7 & 0.29 & 0.13 & -0.18 & 0.26 & -0.39 & 0.45 & -0.60 & 0.40 & -0.31 & 0.30 \\
8 & -0.28 & 0.19 & -0.01 & -0.04 & 0.08 & -0.31 & 0.38 & -0.39 & 0.29 & -0.45 \\
9 & 0.33 & -0.10 & 0.18 & -0.10 & 0.15 & -0.15 & 0.16 & -0.30 & 0.48 & -0.52 \\
10 & -0.23 & 0.12 & -0.20 & 0.18 & -0.12 & 0.17 & -0.12 & 0.25 & -0.36 & 0.32 \\
\hline
\end{tabular}




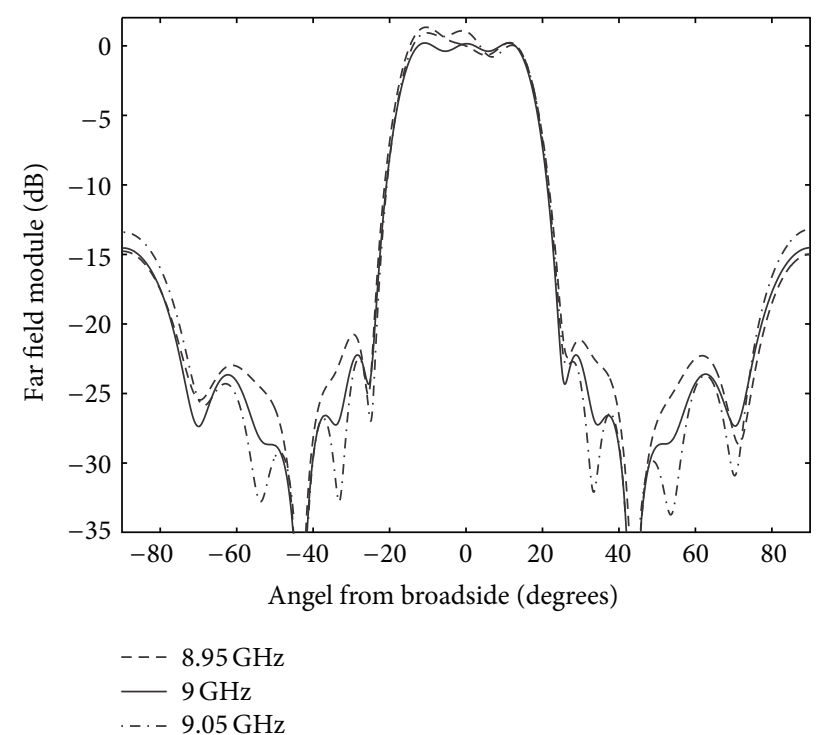

FIGURE 11: Simulated $E$-plane far field of the $8 \times 8$ array with a circular radiation pattern.

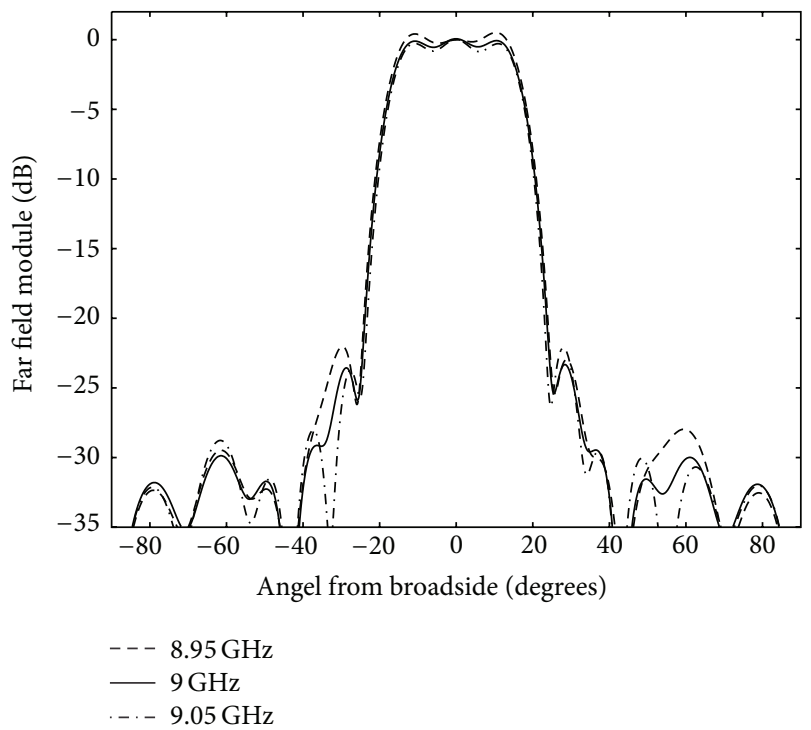

FIGURE 12: Simulated $H$-plane far field of the $8 \times 8$ array with a circular radiation pattern.

the 3D far field pattern is depicted, and Figure 15 shows the difference between the pattern obtained using the required slot voltages and the designed one. In the shaped region, this difference is less than $0.2 \mathrm{~dB}$. Figure 16 shows an enlargement of the shaped region. Finally, the frequency response of the array is shown in Figure 10, while Figures 17 and 18 show, respectively, the $E$-plane and the $H$-plane far field patterns in the array frequency bandwidth. Also in this case, the behaviour of the shaped radiation pattern remains very satisfactory even at the upper and at the lower ends of the useful bandwidth.

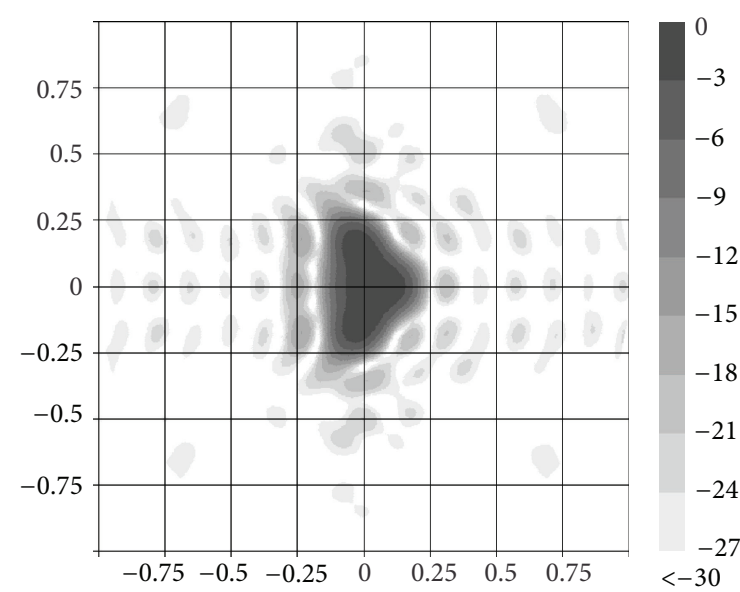

FIGURE 13: Contour plot ( $\mathrm{dB}$ scale) of the $10 \times 10$ planar array with an arrow-shaped radiation pattern in the $u$ - $v$ plane.

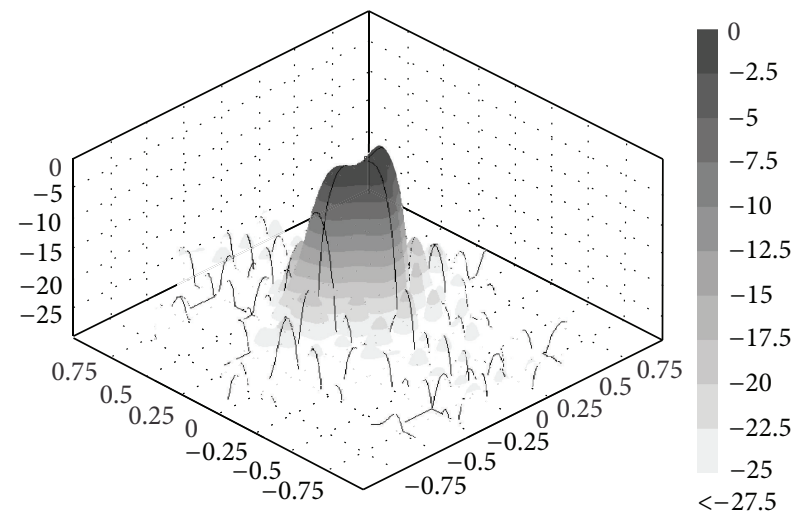

FIGURE 14: 3D radiation pattern (dB scale) of the $10 \times 10$ planar array with an arrow-shaped radiation pattern in the $u$ - $v$ plane.

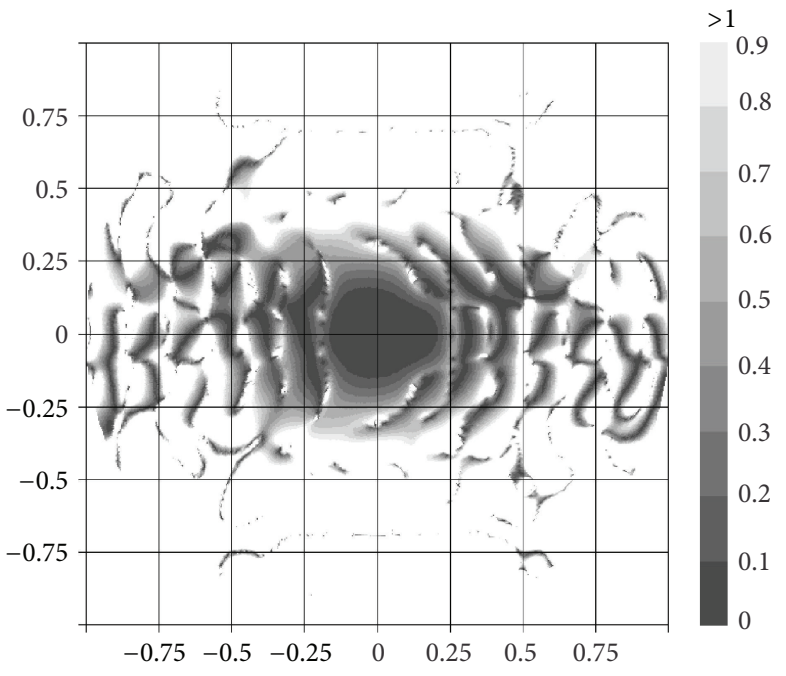

FIGURE 15: Differences between the required and designed radiation pattern (both in $\mathrm{dB}$ scale) for the $10 \times 10$ planar array with an arrowshaped radiation pattern in the $u-v$ plane. 


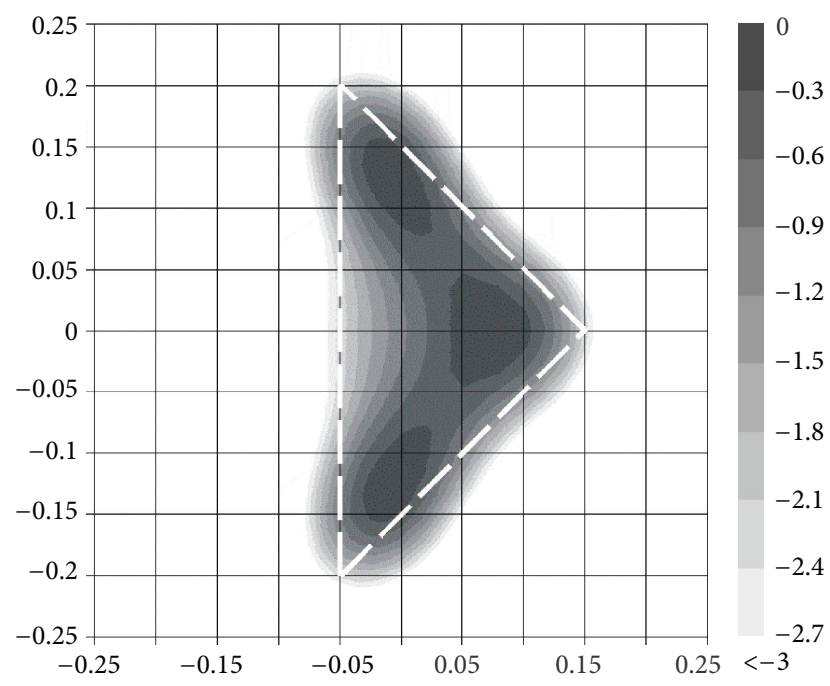

FIGURE 16: Ripple of the $10 \times 10$ planar array with an arrow-shaped radiation pattern in the $u-v$ plane (expressed in $\mathrm{dB}$ ).

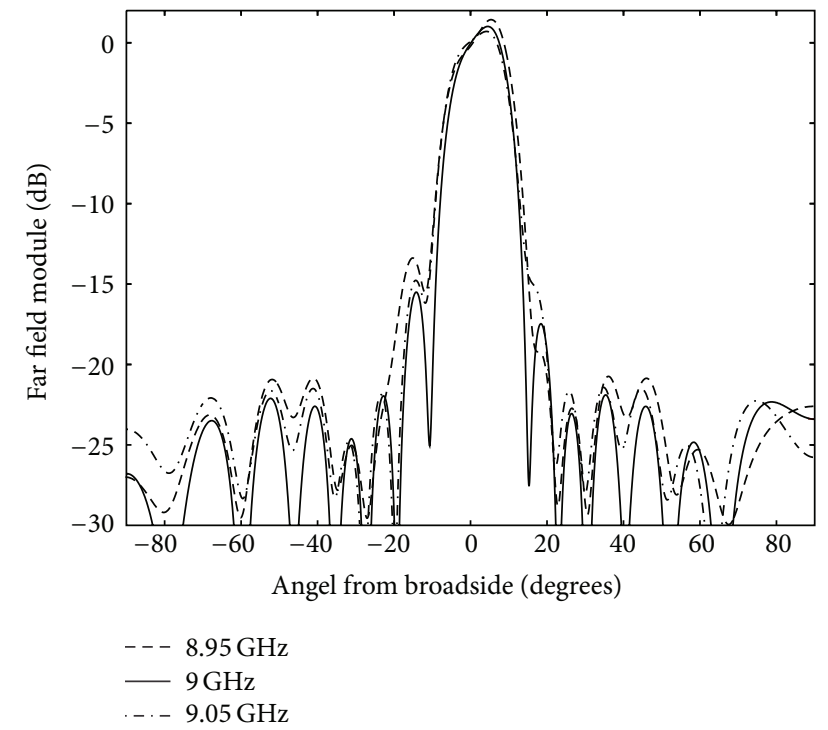

Figure 17: Simulated $E$-plane far field of the $10 \times 10$ array with an arrow-shaped radiation pattern.

The results of the performed simulations, both at the design frequency and within the operating frequency bandwidth of the designed arrays, are in very good agreement with the required specifications, and this fully validates the proposed synthesis procedure.

\section{Conclusion}

The use of shaped beam waveguide slot arrays is required in various antenna applications, such as radar and aerospace applications. A synthesis procedure for shaped beam planar slot arrays has been presented. Starting from the well-known Elliott's model for a (pencil-beam) slot array, an extended set of design equations has been set up to include both the

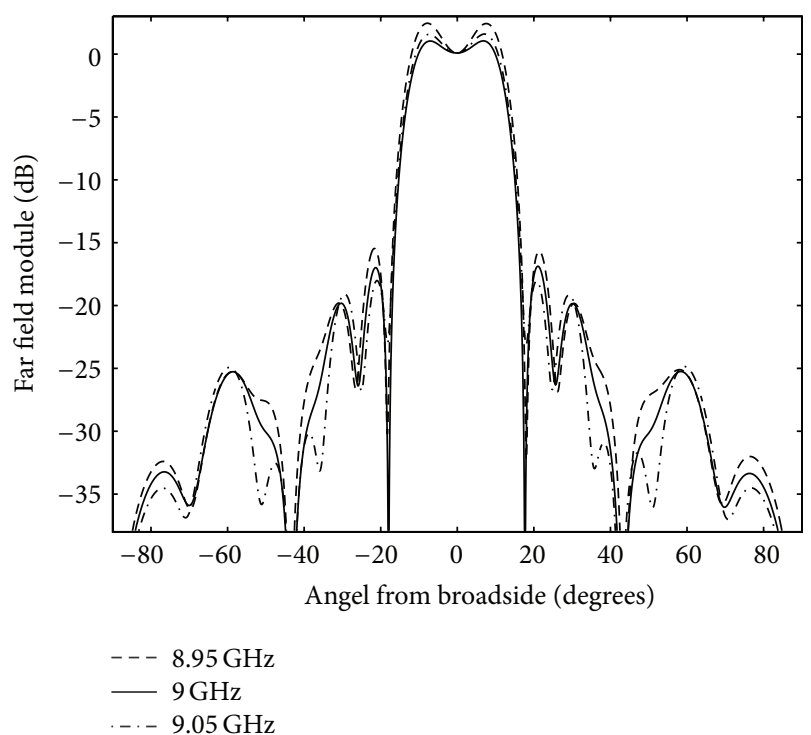

FIGURE 18: Simulated $H$-plane far field of the $10 \times 10$ array with an arrow-shaped radiation pattern.

feeding guide interaction between radiating slots and the provision of complex aperture distribution. Then, a design procedure for shaped beam planar arrays has been devised and assessed through validation against a commercial FEM software.

\section{Acknowledgments}

The authors would like to thank the associate editor professor Sembiam R. Rengarajan and the reviewers of this paper. Their comments have added much to the quality of this paper, helping the authors to clarify out thought.

\section{References}

[1] R. J. Stegen, "Slot radiators and arrays at X-band," IEEE Transactions on Antennas and Propagation, vol. 1, pp. 62-64, 1952.

[2] R. S. Elliott, Antenna Theory and Design, Prentice-Hall, New York, NY, USA, 1981.

[3] S. R. Rengarajan, L. G. Josefsson, and R. S. Elliott, "Waveguidefed slot antennas and arrays: a review," Electromagnetics, vol. 19, no. 1, pp. 3-22, 1999.

[4] G. Montisci, "Design of circularly polarized waveguide slot linear arrays," IEEE Transactions on Antennas and Propagation, vol. 54, no. 10, pp. 3025-3029, 2006.

[5] G. Montisci and G. Mazzarella, "Full-wave analysis of a waveguide printed slot," IEEE Transactions on Antennas and Propagation, vol. 52, no. 8, pp. 2168-2171, 2004.

[6] G. A. Casula, G. Mazzarella, and G. Montisci, "Design of slot arrays in waveguide partially filled with dielectric slab," Electronics Letters, vol. 42, no. 13, pp. 730-731, 2006.

[7] R. C. Johnson and H. Jasik, Antenna Engineering Handbook, McGraw-Hill, New York, NY, USA, 2nd edition, 1984. 
[8] J. Hirokawa, M. Ando, and N. Goto, "Waveguide-fed parallel plate slot array antenna," IEEE Transactions on Antennas and Propagation, vol. 40, no. 2, pp. 218-223, 1992.

[9] J. Hirokawa and M. Ando, "Efficiency of 76-GHz post-wall waveguide-fed parallel-plate slot arrays," IEEE Transactions on Antennas and Propagation, vol. 48, no. 11, pp. 1742-1745, 2000.

[10] S. Park, Y. Tsunemitsu, J. Hirokawa, and M. Ando, "Center feed single layer slotted waveguide array," IEEE Transactions on Antennas and Propagation, vol. 54, no. 5, pp. 1474-1480, 2006.

[11] S. Costanzo, G. A. Casula, A. Borgia et al., "Synthesis of slot arrays on integrated waveguides," IEEE Antennas and Wireless Propagation Letters, vol. 9, pp. 962-965, 2010.

[12] G. A. Casula, G. Mazzarella, and G. Montisci, "A truncated waveguide fed by a microstrip as radiating element for high performance automotive anti-collision radars," International Journal of Antennas and Propagation, vol. 2012, Article ID 983281, 9 pages, 2012.

[13] Z. Jin, G. Montisci, G. Mazzarella, M. Li, H. Yang, and G. A. Casula, "Effect of a multilayer dielectric cover on the behavior of waveguide longitudinal slots," IEEE Antennas and Wireless Propagation Letters, vol. 11, pp. 1190-1193, 2012.

[14] G. Montisci, G. Mazzarella, and G. A. Casula, "Effective analysis of a waveguide longitudinal slot with cavity," IEEE Transactions on Antennas and Propagation, vol. 60, pp. 3104-3110, 2012.

[15] G. A. Casula and G. Montisci, "Design of dielectric-covered planar arrays of longitudinal slots," IEEE Antennas and Wireless Propagation Letters, vol. 8, pp. 752-755, 2009.

[16] R. S. Elliott, "An improved design procedure for small arrays of shunt slots," IEEE Transactions on Antennas and Propagation, vol. 31, no. 1, pp. 48-53, 1983.

[17] R. S. Elliott, The Design of Waveguide-Fed Slot Arrays, edited by Y. T. Lo and S. W . Lee, Van Nostrand, Rheinhold, New York, NY, USA, 1988.

[18] G. Mazzarella and G. Panariello, "Design of slot arrays for SAR applications," Alta Frequenza, vol. 55, no. 6, pp. 359-364, 1986.

[19] IEEE Standard definitions of terms for antennas, IEEE Std 1451993.

[20] R. V. Gatti and R. Sorrentino, "Slotted waveguide antennas with arbitrary radiation pattern," in Proceedings of the 34th European Microwave Conference, pp. 821-824, Amsterdam, The Netherlands, October 2004.

[21] R. V. Gatti, L. Marcaccioli, and R. Sorrentino, "Design of slotted waveguide arrays with arbitrary complex slot voltage distribution," in IEEE Antennas and Propagation Society Symposium, pp. 3265-3268, Monterey, Calif, USA, June 2004.

[22] G. A. Casula, G. Mazzarella, and G. Montisci, "Shaped beam synthesis technique for linear arrays of waveguide longitudinal slots," in Proceedings of the IEEE AP-S International Symposium, Chicago, Ill, USA, 2012.

[23] F. Ares, R. S. Elliott, and E. Moreno, "Synthesis of shaped linesource antenna beams using pure real distributions," Electronics Letters, vol. 30, no. 4, pp. 280-281, 1994.

[24] G. Franceschetti, G. Mazzarella, and G. Panariello, "Array synthesis with excitation constraints," IEE Proceedings $H$, vol. 135 , no. 6, pp. 400-407, 1988.

[25] K. W. Leung and L. Y. Chan, "The probe-fed zonal slot antenna cut onto a cylindrical conducting cavity," IEEE Transactions on Antennas and Propagation, vol. 53, no. 12, pp. 3949-3952, 2005.

[26] S. R. Rengarajan, M. S. Zawadzki, and R. E. Hodges, "Waveguide-slot array antenna designs for low-averagesidelobe specifications," IEEE Antennas and Propagation Magazine, vol. 52, no. 6, pp. 89-98, 2010.
[27] M. Hamadallah, "Frequency limitations on broad-band performance of shunt slot arrays," IEEE Transactions on Antennas and Propagation, vol. 37, no. 7, pp. 817-823, 1989.

[28] G. A. Casula and G. Mazzarella, "A direct computation of the frequency response of planar waveguide slot arrays," IEEE Transactions on Antennas and Propagation, vol. 52, no. 7, pp. 1909-1912, 2004.

[29] J. C. Coetzee, J. Joubert, and D. A. McNamara, "Off-centerfrequency analysis of a complete planar slotted-waveguide array consisting of subarrays," IEEE Transactions on Antennas and Propagation, vol. 48, no. 11, pp. 1746-1755, 2000.

[30] S. R. Rengarajan, "Analysis of a centered-inclined waveguide slot coupler," Transactions on Microwave Theory and Techniques, vol. 37, pp. 884-889, 1989.

[31] R. S. Elliott and W. R. O'Loughlin, "The design of slot arrays including internal mutual coupling," IEEE Transactions on Antennas and Propagation, vol. 34, pp. 1149-1154, 1986.

[32] S. R. Rengarajan and G. M. Shaw, "Accurate characterization of coupling junctions in waveguide-fed planar slot arrays," IEEE Transactions on Microwave Theory and Techniques, vol. 42, no. 12, pp. 2239-2248, 1994.

[33] G. Mazzarella and G. Montisci, "Wideband equivalent circuit of a centered-inclined waveguide slot coupler," Journal of Electromagnetic Waves and Applications, vol. 14, no. 1, pp. 133$151,2000$.

[34] G. A. Casula, G. Mazzarella, and G. Montisci, "Effect of the feeding T-junctions in the performance of planar waveguide slot arrays," IEEE Antennas and Wireless Propagation Letters, vol. 11, pp. 953-956, 2012.

[35] G. Mazzarella and G. Montisci, "Rigorous analysis of dielectriccovered narrow longitudinal shunt slots with finite wall thickness," Electromagnetics, vol. 19, no. 5, pp. 407-418, 1999.

[36] G. Mazzarella and G. Panariello, "On the evaluation of mutual coupling between slots," IEEE Transactions on Antennas and Propagation, vol. 35, no. 11, pp. 1289-1293, 1988.

[37] Z. Jin, G. Montisci, G. A. Casula, H. Yang, and J. Lu, "Efficient evaluation of the external mutual coupling in dielectric covered waveguide slot arrays," International Journal of Antennas and Propagation, vol. 2012, Article ID 491242, 7 pages, 2012. 

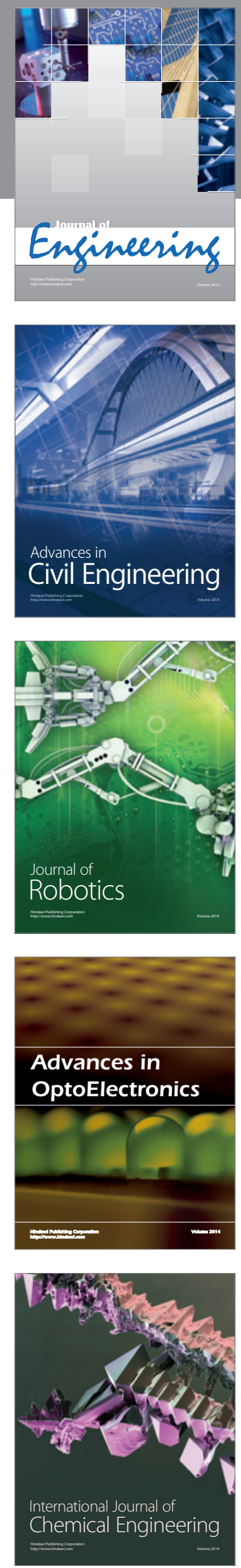

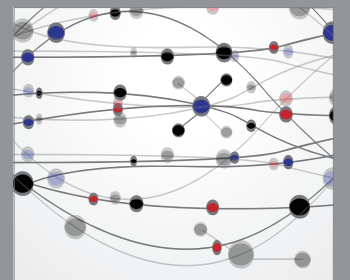

The Scientific World Journal
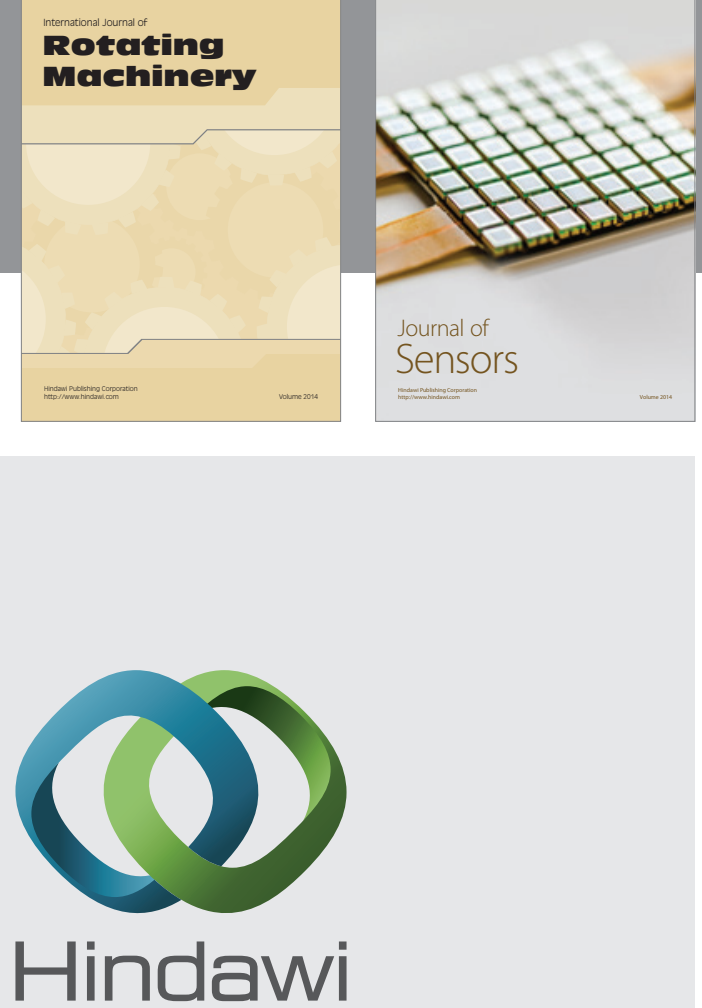

Submit your manuscripts at http://www.hindawi.com
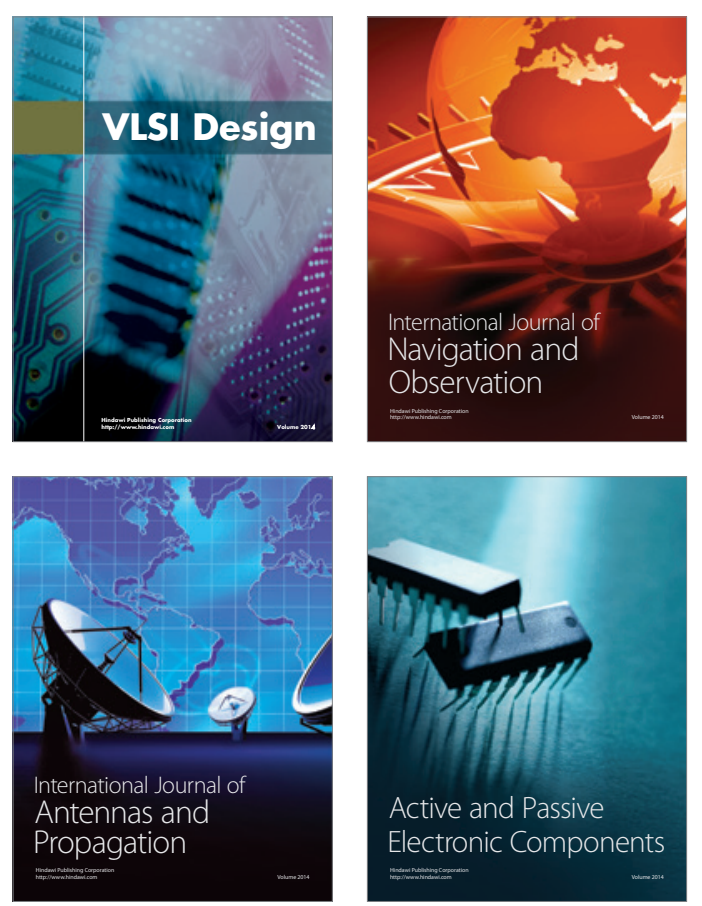
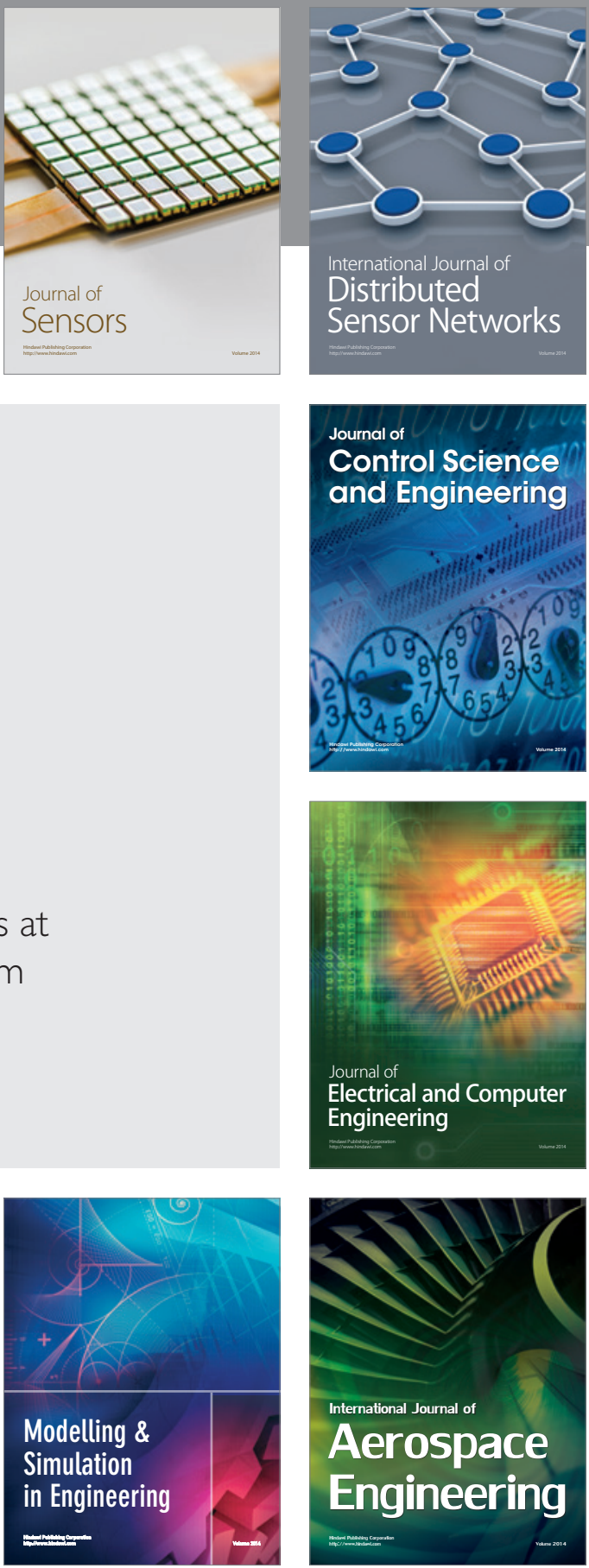

Journal of

Control Science

and Engineering
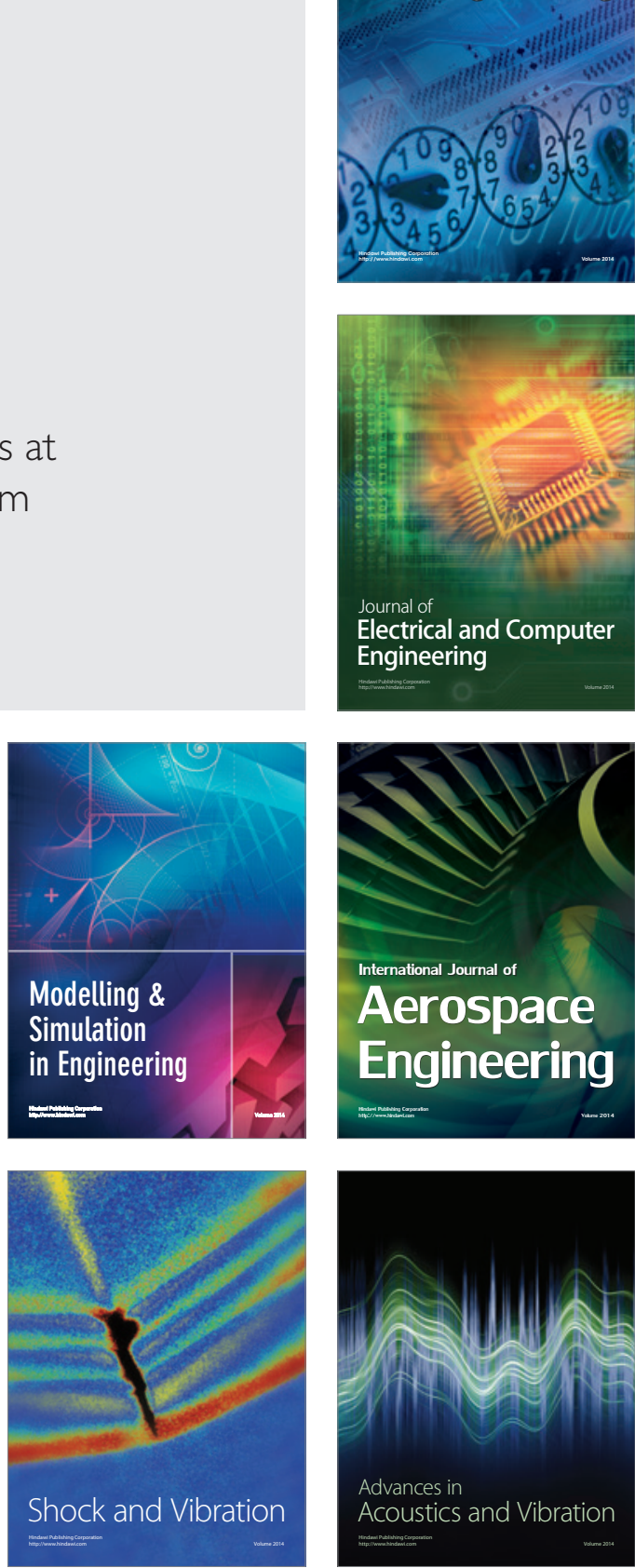